\title{
ON ALGEBRAIC NUMBERS CLOSE TO 1
}

\author{
ARTŪRAS DUBICKaS
}

We prove that there exists a polynomial of small height with a root close to 1 . This implies that there are algebraic numbers close to 1 with relatively small Mahler measure. We also give an explicit construction of such numbers with small Weil height.

\section{INTRODUCTION}

Let $\alpha$ be an algebraic number of degree $d$, with complex conjugates $\alpha_{1}=\alpha, \alpha_{2}, \ldots, \alpha_{d}$ and minimal polynomial $a\left(x-\alpha_{1}\right)\left(x-\alpha_{2}\right) \ldots\left(x-\alpha_{d}\right) \in \mathbb{Z}[x]$, where $a>0$. We denote, as usual, by $M(\alpha)$ Mahler's measure of $\alpha$ and by $h=h(\alpha)$ Weil's height of $\alpha$ :

$$
M(\alpha)=a \prod_{j=1}^{d} \max \left\{1,\left|\alpha_{j}\right|\right\}, \quad h(\alpha)=\frac{\log M(\alpha)}{d}
$$

Suppose that $\alpha \neq 1$. Liouville's inequality gives the following lower bound for $|\alpha-1|$ in terms of $d$ and $M(\alpha)$ :

$$
|\alpha-1| \geqslant 2^{-d+1} M(\alpha)^{-1} \text {. }
$$

The dependence on $M(\alpha)$ in this bound is sharp. For instance, one can take $\alpha=$ $(1-1 / a)^{1 / d}$ with large $a$ (see [12] for similar simple examples). However, this is not the case for the dependence on $d$. For algebraic numbers of small measure and large degree the lower bound for $|\alpha-1|$ can be significantly improved. Mignotte [11] (see also [13, Chapter VII, Section 11]), proved that

$$
|\alpha-1| \geqslant \exp (-4 \sqrt{d} \log (16 d))
$$

provided that $M(\alpha) \leqslant 2$. In the recent years, this inequality has been strengthened by means of Schneider's method and interpolation determinants. We now briefly describe these results.

There is no loss of generality in assuming that $h=h(\alpha)>0$. Indeed, if $h=0$ then, by Kronecker's theorem, $\alpha$ is either 0 or root of unity. Hence, we have the following

\section{Received 23rd March, 1998}

This research was supported by Grant from the Lithuanian Foundation of Studies and Science.

Copyright Clearance Centre, Inc. Serial-fee code: 0004-9729/98 \$A2.00+0.00. 
strong lower bound: $|\alpha-1| \gg(d \log \log d)^{-1}$ (see also [3]). Mignotte and Waldschmidt [12] proved that for each $\varepsilon>0$ there exists $\delta>0$ such that

$$
|\alpha-1|>\exp (-(1+\varepsilon) d \sqrt{h \log (1 / h)})
$$

provided that $0<h<\delta$. This result was strengthened in [7]. Written in the form (1) it gives the constant $\sqrt{2 / 3}+\varepsilon$ instead of $1+\varepsilon$. Finally, the author [9] considered the polynomials

$$
F(x)=F_{k}(x)=\prod_{1 \leqslant v<u \leqslant k}\left(x^{u-v}-1\right)^{J_{u} J_{v}}
$$

where $J_{u}=[k \sin (\pi u / k)]$ and $k \geqslant 3$ is an integer. Taking $k=[\pi / 2 \sqrt{(1 / h) \log (1 / h)}]$ and estimating the product

$$
a^{\mathrm{deg} F} \prod_{j=1}^{d} F\left(\alpha_{j}\right)
$$

we proved that

$$
\begin{aligned}
|\alpha-1| & >\exp \left(-\left(\frac{\pi}{4}+\varepsilon\right) d \sqrt{h \log (1 / h)}\right) \\
& =\exp \left(-\left(\frac{\pi}{4}+\varepsilon\right) \sqrt{d \log M(\alpha) \log (d / \log M(\alpha))}\right)
\end{aligned}
$$

under the same hypotheses.

On the other hand, Amoroso [2] considered the polynomial

$$
G(x)=1+(x+1) \prod_{j=1}^{r}\left(x^{2 j-1}-1\right)
$$

of degree $\mathcal{D}=r^{2}+1$. He showed that there exists a root of $G(-x)$ close to 1

$$
|\alpha-1| \leqslant\left(r^{2}+1\right) 2^{-r}=\exp (-\sqrt{\mathcal{D}-1} \log 2+\log \mathcal{D}),
$$

and that the Mahler measure of $G$ is bounded as follows:

$$
M(G) \leqslant \exp \left(\frac{1}{4 \pi^{2}}(\log \mathcal{D})^{2}+\frac{3}{2} \log \mathcal{D}+7\right)
$$

The minimal polynomial of $\alpha$ divides $G(-x)$, so that its Mahler measure is bounded from above by the same quantity and $d=\operatorname{deg} \alpha \leqslant \mathcal{D}$. It is clear that $\alpha$ is not a root of unity. Thus, we have

$$
|\alpha-1|<\exp (-(2 \pi \log 2-\varepsilon) \sqrt{d \log M(\alpha)} / \log d)
$$

This shows that the inequality (3) is not far from being sharp. Utilising the above example and the results of the work on Lehmer's conjecture (see, for example, [8]) it is not difficult to prove the existence of algebraic numbers for which

$$
|\alpha-1|<\exp (-(2 \pi \log 2-\varepsilon) d \sqrt{h} / \log (1 / h)) .
$$


The aim of this paper is to prove that there are algebraic numbers which are even closer to 1. Siegel's classical result on small solutions of systems of linear equations implies the existence of a polynomial with low height and high vanishing at 1 . We use a modern version of Siegel's lemma due to Bombieri and Vaaler $[5,6]$ in order to construct algebraic numbers close to 1 . We also give an explicit construction of such numbers utilising the polynomial $F(x)$ given by (2).

\section{Statement of the Results}

We recall that the height of a polynomial is the maximum of the absolute values of its coefficients.

THEOREM 1. Let $H$ be a positive integer and let $\varepsilon>0$. For sufficiently large $n$, $n \geqslant n(\varepsilon)$, there is polynomial with integer coefficients of degree at most $n-1$ and of height at most $H$ with a root $\alpha \neq 1$ satisfying

$$
|\alpha-1|<\exp \left(-\left(\frac{4 \log 3}{\sqrt{10}}-\varepsilon\right) \sqrt{\frac{n \log (H+1)}{\log n}}\right) .
$$

Moreover, if $H$ is sufficiently large, $H \geqslant H(\varepsilon)$, then the constant $(4 / \sqrt{10}) \log 3-\varepsilon$ in (6) can by replaced by $2 \sqrt{2 / 7} \log 7-\varepsilon$.

In particular, there exists a polynomial of degree $\leqslant n-1$ with coefficients $0,+1,-1$ which has a root $\alpha, \alpha \neq 1$, close to 1 :

$$
|\alpha-1|<\exp \left(-\left(\frac{4 \log 3 \log 2}{\sqrt{10}}-\varepsilon\right) \sqrt{\frac{n}{\log n}}\right)<\exp \left(-0.9632 \sqrt{\frac{n}{\log n}}\right) .
$$

COROLlaRY. Let $\varepsilon>0$. There exist a constant $c_{1}=c_{1}(\varepsilon)$ and an infinite sequence of positive integers $d$ with the following property: for each $d$ there is an algebraic number $\alpha$ of degree $d$ such that $0<\log M(\alpha)<c_{1} \log d$ and

$$
|\alpha-1|<\exp \left(-\left(\frac{2 \sqrt{2} \log 7}{\sqrt{7}}-\varepsilon\right) \sqrt{\frac{d \log M(\alpha)}{\log d}}\right) .
$$

By Dobrowolski's result [8] we have $\log M(\alpha)>c_{2}(\log d)^{-3}$ whenever $\log M(\alpha)$ is strictly positive. Thus, the Weil height of the above numbers is in the range

$$
\frac{c_{2}}{d(\log d)^{3}}<h<\frac{c_{1} \log d}{d}
$$

so that

$$
|\alpha-1|<\exp \left(-\left(\frac{2 \sqrt{2} \log 7}{\sqrt{7}}-\varepsilon\right) d \sqrt{h / \log (1 / h)}\right)
$$


Clearly, (7) improves (4) by a factor $c_{2} \sqrt{\log d}$ and (8) improves (5) by a factor $c_{3} \sqrt{\log (1 / h)}$. In the following theorem we give an explicit construction of algebraic numbers of small Weil height and close to 1 .

THEOREM 2. Let $\varepsilon>0$, and let $d$ be a sufficiently large positive integer $d \geqslant d(\varepsilon)$. Suppose that $k$ is the largest integer for which $2+4 \operatorname{deg} F_{k} \leqslant d$, where $F=F_{k}$ is given by (2). Then

$$
x^{d}+2(x-1) \frac{F\left(x^{5}\right)}{F(x)}
$$

is an irreducible polynomial of degree $d$ in $\mathbb{Z}[x]$ which has a root $\alpha$ such that

$$
|\alpha-1|<\exp \left(-\left(\frac{4 \log 5}{\pi}-\varepsilon\right) d \sqrt{h / \log (1 / h)}\right) .
$$

Note that numerically one has $(4 / \pi) \log 5=2.0491 \ldots, 2 \sqrt{2 / 7} \log 7=2.0802 \ldots$, hence the inequality (8) is stronger than (9). We shall show below that the Weil height of the algebraic numbers in Theorem 2 is bounded above by $h(\alpha) \leqslant c_{4} d^{-2 / 5} \log d$.

\section{Preliminary Lemmas}

We begin with the following version of Siegel's lemma:

LEMmA 1. Let $H$ be an integer in the range $1 \leqslant H<\exp (N \log \log N)$ and let $\varepsilon>0$. For sufficiently large $N, N \geqslant N(\varepsilon)$, there is a nonzero polynomial $f(x)$ in $\mathbb{Z}[x]$ of degree $\leqslant N-1$ and of height $\leqslant H$ vanishing at 1 with multiplicity at least $[(2-\varepsilon) \sqrt{N \log (H+1) / \log N}]$. Moreover, if $H$ is sufficiently large, $H(\varepsilon) \leqslant H<\exp (N \log \log N)$, then the order of vanishing of $f(x)$ at 1 is at least $[(\sqrt{8}-\varepsilon) \sqrt{N \log (H+1) / \log N}]$.

Suppose that $r<N$ are two positive integers. Bombieri and Vaaler [6] proved that there is a nonzero polynomial in $\mathbb{Z}[x]$ of degree $<N$ vanishing at 1 with multiplicity $\geqslant r$ and such that its height is bounded from above by

$$
\exp \left(\frac{r^{2}}{2(N-r)} \log \left(\frac{e^{3 / 2} N}{4 r}\right)\right)
$$

By taking $r=[(2-\varepsilon) \sqrt{N \log (H+1) / \log N}]$, we see that this is less than $H+1$, so that the height of the polynomial is $\leqslant H$. Similarly, the second part of the lemma follows from [1, Theorem 3].

We denote the norm of a polynomial $Q(x) \in \mathbb{C}[x]$ by

$$
\nu(Q)=\max _{|z| \leqslant 1}|Q(z)| \text {. }
$$

Let also $r(Q)$ be the order of vanishing of $Q(x)$ at 1 . 
LEMma 2. For the polynomial $F_{k}$ given in (2) with the choice $J_{u}=[k \sin (\pi u / k)]$ we have the following asymptotic formulas for $k$ tending to infinity:

$$
\begin{aligned}
\operatorname{deg} F_{k} & =n_{k} \sim \frac{k^{5}}{2 \pi^{2}}, \\
\log \nu\left(F_{k}\right) & \leqslant C \sim \frac{k^{3} \log k}{4}, \\
r\left(F_{k}\right) & =\sum_{1 \leqslant v<u \leqslant k} J_{u} J_{v} \sim \frac{2 k^{4}}{\pi^{2}} .
\end{aligned}
$$

We have proved these asymptotic formulas in [9] (see also [10]). Our final lemma is a simple analytical statement which was also used in [2].

LEMma 3. Suppose $\Phi(x)$ is a polynomial of degree $t$ with complex coefficients such that $\Phi^{\prime}(1) \neq 0$. Then there is a root $\alpha$ of $\Phi$ for which

$$
|\alpha-1| \leqslant t\left|\Phi(1) / \Phi^{\prime}(1)\right| .
$$

Indeed, if $\Phi(1)=0$ then the above statement is trivial. Otherwise,

$$
\begin{aligned}
\left|\frac{\Phi^{\prime}(x)}{\Phi(x)}\right| & =\left|\frac{1}{x-\alpha_{1}}+\frac{1}{x-\alpha_{2}}+\ldots+\frac{1}{x-\alpha_{t}}\right| \leqslant \sum_{j=1}^{t} \frac{1}{\left|x-\alpha_{j}\right|} \\
& \leqslant t / \min _{1 \leqslant j \leqslant t}\left|x-\alpha_{j}\right|
\end{aligned}
$$

and the lemma follows by taking $x=1$.

\section{Proof of Theorem 1}

Suppose first that $H \geqslant 2^{n}$. Let us consider the polynomial $H x^{n-1}-H+1$. For one of its zeros $\alpha=(1-1 / H)^{1 /(n-1)}$ we have

$$
|\alpha-1|<\frac{1}{H+1}<\exp \left(-2.1 \sqrt{\frac{n \log (H+1)}{\log n}}\right)
$$

whenever $n \geqslant 580$ and the theorem follows.

We are left with the non-trivial case $H<2^{n}$. Suppose that $f(x)$ is the polynomial of degree $\leqslant N-1$ and of height $\leqslant H$ from Lemma 1 . We put for brevity $r=r(f)$ and

$$
\theta=\left\{\begin{array}{rll}
1, & \text { if } & f^{(r)}(1) f^{(r+1)}(1) \geqslant 0 \\
-1, & \text { if } & f^{(r)}(1) f^{(r+1)}(1)<0
\end{array}\right.
$$

Let us consider the polynomial

$$
g(x)=\theta x^{N}(x-1) f\left(x^{s}\right)+f(x)
$$


where $s \geqslant 2$ is a fixed integer to be chosen later. Clearly, $g(x)$ is a polynomial of degree $\leqslant N(s+1)-s+1$ and of height $\leqslant H$. We put $n=N(s+1)-s+2$. Then

$$
H<2^{n}<\exp (N \log \log N)
$$

and we can apply Lemma 1:

$$
r=r(f) \geqslant\left[(2-\varepsilon) \sqrt{\frac{N \log (H+1)}{\log N}}\right] .
$$

We now show that $g(x)$ has a root $\alpha, \alpha \neq 1$, for which (6) holds. Indeed, Taylor's formula gives the following expansion:

$$
f(x)=\sum_{j=r}^{N-1} \frac{f^{(j)}(1)}{j !}(x-1)^{j} .
$$

On replacing $x$ by $x^{s}$ we obtain

$$
f\left(x^{s}\right)=\sum_{j=r}^{N-1} \frac{f^{(j)}(1)}{j !}\left(x^{s}-1\right)^{j} .
$$

It follows that

$$
\begin{aligned}
g(x) & =\theta x^{N}(x-1) \sum_{j=r}^{N-1} \frac{f^{(j)}(1)}{j !}\left(x^{s}-1\right)^{j}+\sum_{j=r}^{N-1} \frac{f^{(j)}(1)}{j !}(x-1)^{j} \\
& =(x-1)^{r} \Phi(x) .
\end{aligned}
$$

Here $\Phi(x)$ is a polynomial in $\mathbb{Z}[x]$ for which

$$
\begin{aligned}
\Phi(1) & =\frac{f^{(r)}(1)}{r !}, \\
\Phi^{\prime}(1) & =\frac{\theta s^{r} f^{(r)}(1)}{r !}+\frac{f^{(r+1)}(1)}{(r+1) !} .
\end{aligned}
$$

Taking into account the choice of $\theta$ and the fact that $f^{(r)}(1) \neq 0$, we obtain

$$
\left|\frac{\Phi^{\prime}(1)}{\Phi(1)}\right|=\left|\theta s^{r}+\frac{f^{(r+1)}(1)}{(r+1) f^{(r)}(1)}\right| \geqslant s^{r} .
$$

Since $\Phi(1) \neq 0$ and $\Phi^{\prime}(1) \neq 0$, from Lemma 3 we deduce that $\Phi(x)$, and so $g(x)$, has a root $\alpha, \alpha \neq 1$, for which

$$
|\alpha-1| \leqslant(N(s+1)-s+1-r) s^{-r}<n s^{-r} .
$$

Bounding $r$ from below by (10) and substituting $N=(n+s-2) /(s+1)$, we further find

$$
\begin{aligned}
|\alpha-1| & <\exp \left(-(2-2 \varepsilon) \log s \sqrt{\left.\frac{N \log (H+1)}{\log N}\right)}\right. \\
& <\exp \left(-(2-3 \varepsilon) \frac{\log s}{\sqrt{s+1}} \sqrt{\frac{n \log (H+1)}{\log n}}\right) .
\end{aligned}
$$


The choice $s=9$ completes the proof of (6) with $\varepsilon$ being replaced by $3 \varepsilon$. Hence the result with $\varepsilon$ in (6) carries over if we replace $\varepsilon$ by $\varepsilon / 3$ in Lemma 1.

If $H$ is sufficiently large, $H \geqslant H(\varepsilon)$, then we consider the polynomial $f_{1}(x)$ of degree $\leqslant N-1$ and of height $\leqslant[H / 2]$ from Lemma 1 . Let $\theta_{1}$ be the above $\theta$ with $f$ replaced by $f_{1}$. Then

$$
g_{1}(x)=\theta_{1}(x-1) f_{1}\left(x^{s}\right)+f_{1}(x)
$$

is a polynomial of degree $\leqslant s(N-1)+1$ and of height $\leqslant 2[H / 2] \leqslant H$. Similarly, we can take $n=s(N-1)+2$ and argue as above. The lower bound (10) from Lemma 1 will be replaced by

$$
r=r\left(f_{1}\right) \geqslant\left[(\sqrt{8}-\varepsilon) \sqrt{\left.\frac{N \log ([H / 2]+1)}{\log N}\right]} .\right.
$$

Since $H$ and $N$ are large, instead of (11) we now have

$$
\begin{aligned}
|\alpha-1| & <n s^{-r}<\exp \left(-(\sqrt{8}-2 \varepsilon) \log s \sqrt{\frac{N \log ([H / 2]+1)}{\log N}}\right) \\
& <\exp \left(-(\sqrt{8}-3 \varepsilon) \frac{\log s}{\sqrt{s}} \sqrt{\frac{n \log (H+1)}{\log n}}\right) .
\end{aligned}
$$

The choice $s=7$ completes the proof of Theorem 1 .

\section{Proof of the Corollary}

Let us fix a positive number $c$, and let $n$ be a sufficiently large positive integer. We consider the polynomial of degree at most $n-1$ and of height at most $H=\left[n^{c}\right]$ with a root close to 1 as in the second part of Theorem 1 (for example, $g_{1}(x)$ given by (12)). Since $\log (H+1) \geqslant c \log n$, by Theorem 1 the polynomial $g_{1}$ has a root $\alpha \neq 1$ for which

$$
|\alpha-1|<\exp \left(-\left(\frac{2 \sqrt{2} \log 7}{\sqrt{7}}-\varepsilon\right) \sqrt{c n}\right) \text {. }
$$

We write

$$
g_{1}(x)=(x-1)^{r} p(x) g_{2}(x)=\sum_{j=0}^{n-1} b_{j} x^{j}
$$

where $p(x)$ is the minimal polynomial for $\alpha$. Utilising Landau's inequality and the multiplicativity of Mahler's measure we have

$$
M(\alpha)=M(p) \leqslant M\left(g_{1}\right) \leqslant \sqrt{\sum_{j=0}^{n-1}\left|b_{j}\right|^{2}} \leqslant n^{c+1 / 2} .
$$

Clearly, $\alpha$ is not a root of unity, since otherwise the inequality opposite to (13) holds. Thus,

$$
0<\log M(\alpha) \leqslant\left(c+\frac{1}{2}\right) \log n
$$


Now (13) and the trivial bound $d=\operatorname{deg} \alpha<n$ give

$$
\begin{aligned}
|\alpha-1| & <\exp \left(-\left(\frac{2 \sqrt{2} \log 7}{\sqrt{7}}-\varepsilon\right) \sqrt{\frac{c n \log M(\alpha)}{(c+1 / 2) \log n}}\right) \\
& <\exp \left(-\left(\frac{2 \sqrt{2} \log 7}{\sqrt{7}}-2 \varepsilon\right) \sqrt{\frac{d \log M(\alpha)}{\log d}}\right)
\end{aligned}
$$

for sufficiently large $c$.

On the other hand, by Liouville's inequality we have

$$
|\alpha-1| \geqslant 2^{-d+1} M(\alpha)^{-1}>\exp \left(-d \log 2-\left(c+\frac{1}{2}\right) \log n\right) .
$$

Combining this inequality with (13) we see that $d$ is bounded from below as $d>c_{5} \sqrt{c n}$. Thus, there is an infinite sequence of $d$ such as required and

$$
\log M(\alpha) \leqslant\left(c+\frac{1}{2}\right) \log n<c_{1} \log d
$$

This completes the proof.

\section{Proof of Theorem 2}

It is clear that the polynomial

$$
\begin{aligned}
R(x) & =x^{d}+2(x-1) \frac{F\left(x^{5}\right)}{F(x)} \\
& =x^{d}+2(x-1) \prod_{1 \leqslant v<u \leqslant k}\left(1+x^{u-v}+x^{2(u-v)}+x^{3(u-v)}+x^{4(u-v)}\right)^{J_{u} J_{v}}
\end{aligned}
$$

is monic and irreducible, by Eisenstein's criterion. Further, we have that $R(1)=1$ and

$$
R^{\prime}(1)=d+2 \prod_{1 \leqslant v<u \leqslant k} 5^{J_{u} J_{v}}=d+2 \cdot 5^{r(F)}
$$

By Lemma 3, there is a root $\alpha$ of $R(x)$ for which

$$
|\alpha-1|<d 5^{-r(F)} .
$$

The choice of $k$ is such that

$$
2+4 \operatorname{deg} F_{k}=2+4 n_{k} \leqslant d<2+4 n_{k+1} \text {. }
$$

Hence, Lemma 2 implies that $d \sim 2 k^{5} / \pi^{2}$. Let us put

$$
s(F)=s\left(F_{k}\right)=\frac{\log \nu\left(F_{k}\right)}{\operatorname{deg} F_{k}}=\frac{\log \nu\left(F_{k}\right)}{n_{k}} .
$$


From Lemma 2 (see also [10, Theorem 3]) it follows that

$$
r\left(F_{k}\right)>\left(\frac{8}{\pi}-\varepsilon\right) n_{k} \sqrt{s\left(F_{k}\right) / \log \left(1 / s\left(F_{k}\right)\right)} .
$$

Hence, from (14) we see that

$$
|\alpha-1|<\exp \left(-\left(\frac{2 \log 5}{\pi}-2 \varepsilon\right) d \sqrt{s\left(F_{k}\right) / \log \left(1 / s\left(F_{k}\right)\right)}\right) .
$$

Further, $F(x)$ is a product of cyclotomic polynomials. Therefore

$$
M(\alpha)=M(R)=M(R F) \leqslant \nu(R F)=\nu\left(x^{d} F(x)+2(x-1) F\left(x^{5}\right)\right) \leqslant 5 \nu\left(F_{k}\right),
$$

so that we can bound $h(\alpha)$ from above:

$$
h=h(\alpha)=\frac{\log M(\alpha)}{d} \leqslant \frac{\log \left(5 \nu\left(F_{k}\right)\right)}{d}<\left(\frac{1}{4}+\varepsilon\right) s\left(F_{k}\right) .
$$

Theorem 2 now clearly follows from (15). Note that we also have

$$
h<\left(\frac{1}{20}\left(\frac{\pi^{2}}{2}\right)^{3 / 5}+\varepsilon\right) d^{-2 / 5} \log d .
$$

\section{CONCLUding REMARKS}

Recently, Bugeaud [4] considered the norm of $\alpha-1$. He showed [4, Theorem 2] that if $\alpha$ is not a root of unity then for any $\varepsilon>0$, there exists a $\delta>0$, depending only on $\varepsilon$, such that if $\log M(\alpha) \leqslant \delta d, d=\operatorname{deg} \alpha$, then

$$
\log |N(\alpha-1)| \leqslant \sqrt{(2 / 3+\varepsilon) d \log d \log M(\alpha)} .
$$

Moreover, using the example of algebraic numbers close to 1 due to Amoroso [2] he showed $\left[4\right.$, Theorem 3] that the dependence on $d$ in (16) cannot be relaxed to $\sqrt{d} /(\log d)^{1+\varepsilon}$, for any $\varepsilon>0$.

Note that by considering polynomials $(2)$ with $k=[(\pi / 2) \sqrt{(1 / h) \log (1 / h)}]$ from our estimates in [9, Section 3], we get

$$
\log |N(\alpha-1)|<\left(\frac{\pi}{4}+\frac{\varepsilon}{2}\right) d \sqrt{h \log (1 / h)}<\left(\frac{\pi}{4}+\varepsilon\right) \sqrt{d \log d \log M(\alpha)}
$$

under the same hypotheses as in (16). Clearly, this inequality strengthens (16).

On the other hand, let us consider the polynomial

$$
T(x)=x^{d}+2 \frac{F\left(x^{5}\right)}{F(x)}=x^{d}+2 \prod_{1 \leqslant v<u \leqslant k}\left(1+x^{u-v}+x^{2(u-v)}+x^{3(u-v)}+x^{4(u-v)}\right)^{J_{u} J_{v}} .
$$


As in Section 6 we see that $T(x)$ is monic and irreducible. Suppose $\alpha$ is a root of $T(x)$. Then we have

$$
|N(\alpha-1)|=|T(1)|=1+2 \cdot 5^{r(F)}>5^{r(F)} .
$$

From the results of Section 6 we obtain the lower bound for the norm:

$$
\log |N(\alpha-1)|>\left(\frac{4 \log 5}{\pi}-\varepsilon\right) d \sqrt{h / \log (1 / h)} .
$$

This shows that the dependence on $d$ in (16) and (17) cannot be relaxed to $\sqrt{d} /(\log d)^{1 / 2+\varepsilon}$, for any $\varepsilon>0$.

It is easily seen from our arguments in Section 4 that the upper bound for $|\alpha-1|$ depends on the results concerning the order of vanishing $r$ of polynomials at 1 . On the other hand, we remark that the results on the lower bound for $|\alpha-1|$ imply the upper bounds for $r$. An old result of Schur [14] on the number of real roots of a polynomial gives the following bound:

$$
r(f) \leqslant 2 \sqrt{N \log L(f)}
$$

Here $f$ is a polynomial in $\mathbb{Z}[x]$ of degree $<N$ and of length $L(f)$. Recently, Amoroso [1] strengthened the constant in this upper bound

$$
r(f) \leqslant 1.21 \sqrt{N \log L(f)}
$$

provided that $N \rightarrow \infty, \log N / \log L(f) \rightarrow 0$ and $(\log L(f)) / N \rightarrow 0$.

Let us consider the polynomial

$$
g_{3}(x)=\theta(x-1) f\left(x^{s}\right)+f(x),
$$

where $\theta$ is as in Section 4. Analogously, we deduce that $g_{3}(x)$ has a root $\alpha, \alpha \neq 1$, such that

$$
|\alpha-1|<s^{-r(f)} \operatorname{deg} g_{3}<N s^{-r(f)+1} .
$$

In this way we find that

$$
r(f) \log s<-\log |\alpha-1|+\log (N s) .
$$

We see that a lower bound for $|\alpha-1|$ implies an upper bound for $r(f)$. We cannot improve upon (18) utilising the lower bound (3). However, if, for example, the lower bound

$$
|\alpha-1|>\exp \left(-c(\sigma) \sqrt{d \log M(\alpha)}(\log d)^{-\sigma}\right)
$$

holds with $\sigma$ in the range $0 \leqslant \sigma \leqslant 1 / 2$ and constant $c(\sigma)>0$, then

$$
r(f) \log s<c(\sigma) \sqrt{d \log M(\alpha)}(\log d)^{-\sigma}+\log (N s) .
$$

Since $d<N s$ and

$$
M(\alpha) \leqslant M\left(g_{3}\right) \leqslant \nu\left(g_{3}\right) \leqslant 3 \nu(f) \leqslant 3 L(f)
$$


the choice of $s=7$ would further imply

$$
r(f)<1.36 c(\sigma) \sqrt{N \log (3 L(f))}(\log N)^{-\sigma}
$$

for sufficiently large $N$.

Finally, we have noticed in [9] that the lower bound for the difference between $\alpha$ and a root of unity

$$
\left|\alpha-\exp \left(\frac{2 \pi i j}{m}\right)\right|>\exp (-(\pi \sqrt{m} / 8+\varepsilon) d \sqrt{h \log (1 / h)})
$$

where $m \geqslant 3$ is a fixed integer, holds. Replacing the polynomial $g_{1}(x)$ in (12) by $g_{1}\left(x^{m}\right)$ gives an example of algebraic numbers close to $m$-th root of unity. The inequality (see also (8))

$$
\left|\alpha-\exp \left(\frac{2 \pi i j}{m}\right)\right|<\exp \left(-\left(\frac{2 \sqrt{2} \log 7}{\sqrt{7 m}}-\varepsilon\right) d \sqrt{h / \log (1 / h)}\right)
$$

holds for infinite number of $\alpha$.

\section{REFERENCES}

[1] F. Amoroso, 'Polynomials with prescribed vanishing at roots of unity', Boll. Un. Mat. Ital. 7 (1995), 1021-1042.

[2] F. Amoroso, 'Algebraic numbers close to 1 and variants of Mahler's measure', J. Number Theory 60 (1996), 80-96.

[3] F. Amoroso, 'Algebraic numbers close to 1: results and methods', in Number theory, (V.K. Murthy and M. Waldschmidt, Editors), Contemporary Mathematics 210 (American Mathematical Society, Berlin, Heidelberg, New York, 1997), pp. 305-316.

[4] Y. Bugeaud, 'Algebraic numbers close to 1 in non-archimedean metrics', J. Ramanujan Math. Soc. (to appear).

[5] E. Bombieri and J.D. Vaaler, 'On Siegel's lemma', Invent. Math. 73 (1983), 11-32.

[6] E. Bombieri and J.D. Vaaler, 'Polynomials with low height and prescribed vanishing', in Analytic number theory and diophantine problems, (A.C. Adolphson, J.B. Conrey, A. Ghosh and R.I. Yager, Editors), Progress in Mathematics Series 70 (Birkhäuser, Basel, 1987), pp. 53-73.

[7] Y. Bugeaud, M. Mignotte and F. Normandin, 'Nombres algébriques de petite mesure et formes linéaires en un logarithme', C.R. Acad. Sci. Paris Sér. I. 321 (1995), 517-522.

[8] E. Dobrowolski, 'On a question of Lehmer and the number of irreducible factors of a polynomial', Acta Arith. 34 (1979), 391-401.

[9] A. Dubickas, 'On algebraic numbers small measure', Lithuanian Math. J. 35 (1995), 333-342.

[10] A. Dubickas, 'On a polynomial with large number of irreducible factors', in Proceedings of the International Number Theory Conference dedicated to Professor A. Schinzel on the occasion of his 60th birthday, (to appear).

[11] M. Mignotte, 'Approximation des nombres algébriques par des nombres algébriques de grand degré', Ann. Fac. Sci. Toulouse Math. 1 (1979), 165-170. 
[12] M. Mignotte and M. Waldschmidt, 'On algebraic numbers of small height: linear forms in one logarithm', J. Number Theory 47 (1994), 43-62.

[13] W.M. Schmidt, Diophantine approximation, Lecture Notes in Mathematics 785 (Springer-Verlag, Berlin, Heidelberg, New York, 1980).

[14] I. Schur, 'Untersuchungen über algebraische Gleichungen. I. Bemerkungen zu einem Satz von E. Schmidt.', S.-B. preuss. Acad. Wiss. H. 7/10 (1933), 403-428.

Faculty of Mathematics

Vilnius University

Naugarduko 24

Vilnius 2006

Lithuania

e-mail: arturas.dubickas@maf.vu.lt 